\title{
Nutrição enteral em idosos com demência em cuidados paliativos
}

Paula Abrantes Ciccarelli ${ }^{1}$, Emanuela Bezerra Torres Mattos ${ }^{1}$

1. Universidade Federal de São Paulo, Santos/SP, Brasil.

\begin{abstract}
Resumo
O número de idosos e, consequentemente, a incidência de doenças crônicas não transmissíveis, entre elas a demência, têm aumentado exponencialmente. A demência leva a perda progressiva de funcionalidade, incluindo complicações relacionadas com alimentação. Este artigo traz resultados de revisão integrativa sobre as evidências científicas atuais da terapia nutricional enteral em pacientes com demência em cuidados paliativos. Foram incluídos artigos publicados entre 2009 e 2019. Os dados foram coletados em novembro de 2019, em cinco indexadores, a partir de busca pelos descritores "nutrição enteral", "cuidados paliativos" e "demência". Depois de aplicados os critérios de inclusão, 11 artigos compuseram a amostra. Os principais achados dessas publicações foram divididos em três categorias: "cuidados paliativos e plano de cuidados", "cuidados paliativos e nutrição enteral" e "nutrição enteral em idosos com demência". Os resultados chamam atenção para a necessidade de que a tomada de decisão se baseie em evidências científicas.
\end{abstract}

Palavras-chave: Demência. Cuidados paliativos. Nutrição enteral.

\section{Resumen}

\section{Nutrición enteral en ancianos con demencia en cuidados paliativos}

El número de ancianos y, en consecuencia, la incidencia de enfermedades crónicas no transmisibles, entre ellas la demencia, han aumentado exponencialmente. La demencia lleva a una pérdida progresiva de funcionalidad, incluidas complicaciones relacionadas con la alimentación. Este artículo presenta resultados de una revisión integrativa de las evidencias científicas actuales de la terapia nutricional enteral en pacientes con demencia en cuidados paliativos. Para ello, se incluyeron artículos publicados entre el 2009 y el 2019. Los datos se recopilaron en noviembre del 2019, en cinco indexadores, con base en los descriptores "nutrición enteral", "cuidados paliativos" y "demencia". Después de aplicados los criterios de inclusión, la muestra final consistió en 11 artículos. Los principales hallazgos de estas publicaciones se dividieron en tres categorías: "cuidados paliativos y plan de cuidados", "cuidados paliativos y nutrición enteral" y "nutrición enteral en ancianos con demencia". Los resultados Ilaman I

Palabras clave: Demencia. Cuidados paliativos. Nutrición enteral.

\section{Abstract \\ Enteral nutrition in older adults with dementia in palliative care}

The number of older adults and, consequently, the incidence of chronic non-communicable diseases, including dementia, have increased exponentially. Dementia leads to progressive loss of functionality, including food-related complications. This article presents results of an integrative review on the current scientific evidence of enteral nutritional therapy in patients with dementia in palliative care. Articles published between 2009 and 2019 were included. Data were collected in November 2019 in five databases, based on the following search words: "enteral nutrition", "palliative care" and "dementia". After applying the inclusion criteria, 11 articles remained in the sample. The main findings of these publications were divided into three categories: "palliative care and care plan", "palliative care and enteral nutrition" and "enteral nutrition in older adults with dementia". The results call attention to the need for decision making to be based on scientific evidence.

Keywords: Dementia. Palliative care. Enteral nutrition. 
Atualmente o Brasil passa por uma intensa transição demográfica, com o aumento rápido e exponencial do número de idosos em nossa população. Podemos atribuir esse fenômeno a diversos aspectos, como diminuição da natalidade e da mortalidade infantil, aumento da expectativa de vida advindo dos avanços médicos e científicos, ampliação do acesso aos serviços de saúde e menor incidência de doenças infectocontagiosas. Em contrapartida, o aumento das doenças crônicas não transmissíveis, entre elas as demências, marca a mudança na saúde pública brasileira ${ }^{1,2}$.

As síndromes demenciais são caracterizadas pela presença de déficit progressivo na função cognitiva, comprometendo diversas funções cerebrais, incluindo memória, raciocínio, orientação, compreensão e capacidade de aprendizagem, linguagem e julgamento. Em razão da perda acentuada de memória, a demência é uma doença crônica altamente incapacitante. Com sua progressão, o indivíduo se torna gradualmente dependente para atividades de vida diáriaa ${ }^{1,3}$.

A demência tem diversas causas, algumas reversíveis e outras irreversíveis. A mais comum é a doença de Alzheimer, responsável por algo entre $60 \%$ e $70 \%$ dos $\operatorname{casos}^{1}$. De acordo com a OMS ${ }^{4}$, a demência afeta 47 milhões de pessoas no mundo, e estima-se que sua incidência cresça até atingir 75 milhões de pessoas em 2030 e 132 milhões em 2050. Projeções para a população brasileira apontam um crescimento na taxa de prevalência, na população com 65 anos ou mais, de $7,6 \%$ a $7,9 \%$ entre 2010 e 2020 , ou seja, 55.000 novos casos por ano ${ }^{1}$.

Escalas para avaliar a funcionalidade são frequentemente usadas para definir o melhor plano de cuidados para cada paciente. A mais usada e validada em português é a Functional Assessment Staging (Fast), que varia entre estágios designados com números de um (nenhuma dificuldade objetiva ou subjetiva) a sete (paciente em estado grave, já apresentando problemas como disfagia, lesões por pressão e sepse) ${ }^{5}$.

Aproximadamente $90 \%$ dos pacientes com demência vão desenvolver problemas relacionados a alimentação, como disfagia, incapacidade de comer sozinho e recusa alimentar. A disfagia é qualquer dificuldade na condução do alimento da boca até o estômago causada por alterações na dinâmica da deglutição. Ela é o motivo mais comum para instituir a terapia nutricional enteral, principalmente em ambiente hospitalar, por conta do risco de complicações ${ }^{5,6}$. Assim, há indicação dessa terapia para pacientes idosos, com disfagia grave de origem neurológica, para assegurar o suprimento nutricional, além de manter e melhorar o estado nutricional ${ }^{7}$.

Utilizam-se artifícios com suplementação nutricional oral ou nutrição enteral, procedimento pelo qual se administra uma solução de nutrientes por sondas ou via oral. A nutrição enteral é utilizada exclusiva ou parcialmente para substituir ou complementar a alimentação oral em pacientes desnutridos ou não, conforme suas necessidades nutricionais, em regime hospitalar, ambulatorial ou domiciliar. O procedimento visa à síntese ou manutenção de tecidos, órgãos ou sistemas e é recomendado quando a ingestão oral é insuficiente $(<60 \%$ das necessidades nutricionais ideais), quando há doenças do trato gastrointestinal e que impedem a alimentação via oral, e quando se observam alterações do nível de consciência ou deglutição comprometida de causa muscular ou neurológica. O objetivo é garantir a oferta adequada de calorias e de macro e micronutrientes que mantêm o metabolismo, evitando os efeitos deletérios da desnutrição e da sarcopenia, como maior fragilidade, perda da performance física, piora do declínio cognitivo, aumento das taxas de infecções e mortalidade ${ }^{7-11}$.

Todavia, em cada doença e em cada um de seus estágios, é importante analisar riscos e benefícios da terapia nutricional, considerando princípios éticos na tomada de decisão, como autonomia do paciente e beneficência. Quando o quadro nutricional se torna irreversível, a abordagem paliativa deve ser uma alternativa ${ }^{12}$. Nos casos de pacientes que desenvolvem doenças graves, progressivas e incuráveis, como a demência, os cuidados paliativos deveriam ser iniciados desde o diagnóstico ${ }^{13}$.

Os cuidados paliativos visam melhorar a qualidade de vida dos pacientes e de seus familiares, aliviando a dor e outros sintomas que causam sofrimento. Tais cuidados, focados na pessoa, e não na doença, são a única terapêutica cabível durante o processo ativo de morte. Na demência, além do foco na qualidade de vida, manter a funcionalidade e maximizar o conforto são objetivos da assistência em toda a trajetória da doença ${ }^{5}$.

$\mathrm{O}$ ato de se alimentar representa muito mais do que a ingestão de calorias e nutrientes. Ele está diretamente relacionado com aspectos emocionais, 
socioculturais, religiosos e com experiências vividas ao longo da vida. É difícil entender o que leva o paciente a perder peso - algo comum entre pacientes em cuidados paliativos - sem conversar com familiares ou com responsáveis pelos cuidados, que sofrem e sentem angústia diante dessa situação. Por esse motivo, é necessário conhecer os desejos do paciente e de sua família, considerando os objetivos do cuidado e o estágio da doença ${ }^{5,12}$.

As diretivas antecipadas de vontade, pautadas no princípio bioético da autonomia, permitem que a pessoa registre sua vontade caso uma doença se agrave e ela não possa mais responder por si mesma. Esse instrumento, além de servir de suporte ético e legal para profissionais da saúde, visa respeitar a vontade do paciente, mantendo-o protagonista de sua própria vida ${ }^{13}$. O conhecimento da demência pelo paciente e seus familiares - isto é, entender que se trata de doença terminal, e é preciso priorizar a qualidade de vida -, Ihes dá a oportunidade de se preparar para a proposta de final de vida e tomada de decisões ${ }^{5}$.

O presente artigo apresenta os objetivos do uso da terapia nutricional enteral em pacientes com demência em cuidados paliativos de acordo com os diferentes momentos da doença, abordando os benefícios e malefícios dessa terapia, bem como os aspectos éticos e as evidências científicas atuais sobre o tema. Para isso, o texto traz resultados de uma revisão integrativa da literatura dos últimos dez anos sobre nutrição enteral em pacientes com demência em cuidados paliativos.

\section{Método}

Para elaborar o estudo, utilizou-se o método de revisão integrativa, que permite incorporar evidências na prática clínica, sintetizando o conhecimento científico já produzido sobre o tema investigado. Para isso, foram cumpridas as seguintes etapas: 1) elaboração da pergunta norteadora; 2) estabelecimento dos critérios de inclusão e de exclusão; 3) definição dos descritores, busca na literatura e coleta de dados; 4) análise crítica dos estudos incluídos e discussão dos resultados; e 5) síntese do conhecimento produzido ${ }^{14-16}$.

A pergunta norteadora definida foi: "Qual o conhecimento científico produzido na literatura sobre a utilização de terapia nutricional enteral em pacientes com demência em cuidados paliativos?". Estabeleceram-se como critérios de inclusão: estudos publicados nos últimos 10 anos (entre 2009 e 2019), escritos em português, inglês ou espanhol, disponíveis na íntegra, e que abordassem nutrição enteral, cuidados paliativos e demência. Foram excluídos estudos que abordavam a nutrição parenteral e que tratavam apenas de doenças específicas, como o câncer.

A coleta de dados foi realizada em novembro de 2019, nas bases de dados Scientific Electronic Library Online (SciELO), Literatura Latino-Americana e do Caribe em Ciências da Saúde (Lilacs), Periódicos Capes, Cochrane e PubMed. Foram utilizadas na pesquisa as seguintes palavras-chave, indexadas aos Descritores em Ciências da Saúde (DeCS) e ao Medical Subject Headings (MeSH), em português e inglês: "nutrição enteral", "cuidados paliativos" e "demência" - "enteral nutrition", "palliative care" e "dementia". Como estratégia de busca, os descritores foram combinados por meio dos operadores booleanos and e or.

A análise dos artigos foi feita primeiramente por meio da leitura dos títulos, seguida da leitura dos resumos e, posteriormente, da leitura do texto na íntegra, além de interpretação crítica dos artigos selecionados por meio de discussão entre as pesquisadoras.

\section{Resultados}

A busca resultou em 30 artigos encontrados nas seguintes bases de dados: PubMed $(n=21)$, Cochrane $(n=4)$, SciELO $(n=3)$, Periódicos Capes $(n=1)$ e Lilacs $(n=1)$. Após leitura dos títulos e resumos, com aplicação dos critérios de inclusão, permaneceram 15 artigos, que foram lidos na íntegra. Depois dessa leitura, 11 artigos se mantiveram na seleção final (Figura 1).

Os estudos incluídos foram publicados entre 2009 e 2019: 3 artigos em 2010, 2 em 2014, 2 em 2017, e os restantes (4 artigos) cada um em um ano diferente (2009, 2012, 2015 e 2016). Nos outros anos considerados, não foram encontradas produções que cumprissem os critérios de inclusão. Quanto ao local de origem, a maioria das pesquisas foi feita nos Estados Unidos (36,4\%), seguido de Reino Unido (27,3\%), Brasil (18,2\%), Singapura e Chile (ambos com 9,1\%). Quanto ao idioma, 
$72,7 \%$ dos artigos foram publicados em língua inglesa, 18,2\% em português, e 9,1\% em espanhol. O Quadro 1 apresenta dados dos estudos selecionados: título, autores, ano de publicação, país de origem, idioma, método empregado e principais objetivos.

Figura 1. Fluxograma de seleção dos artigos

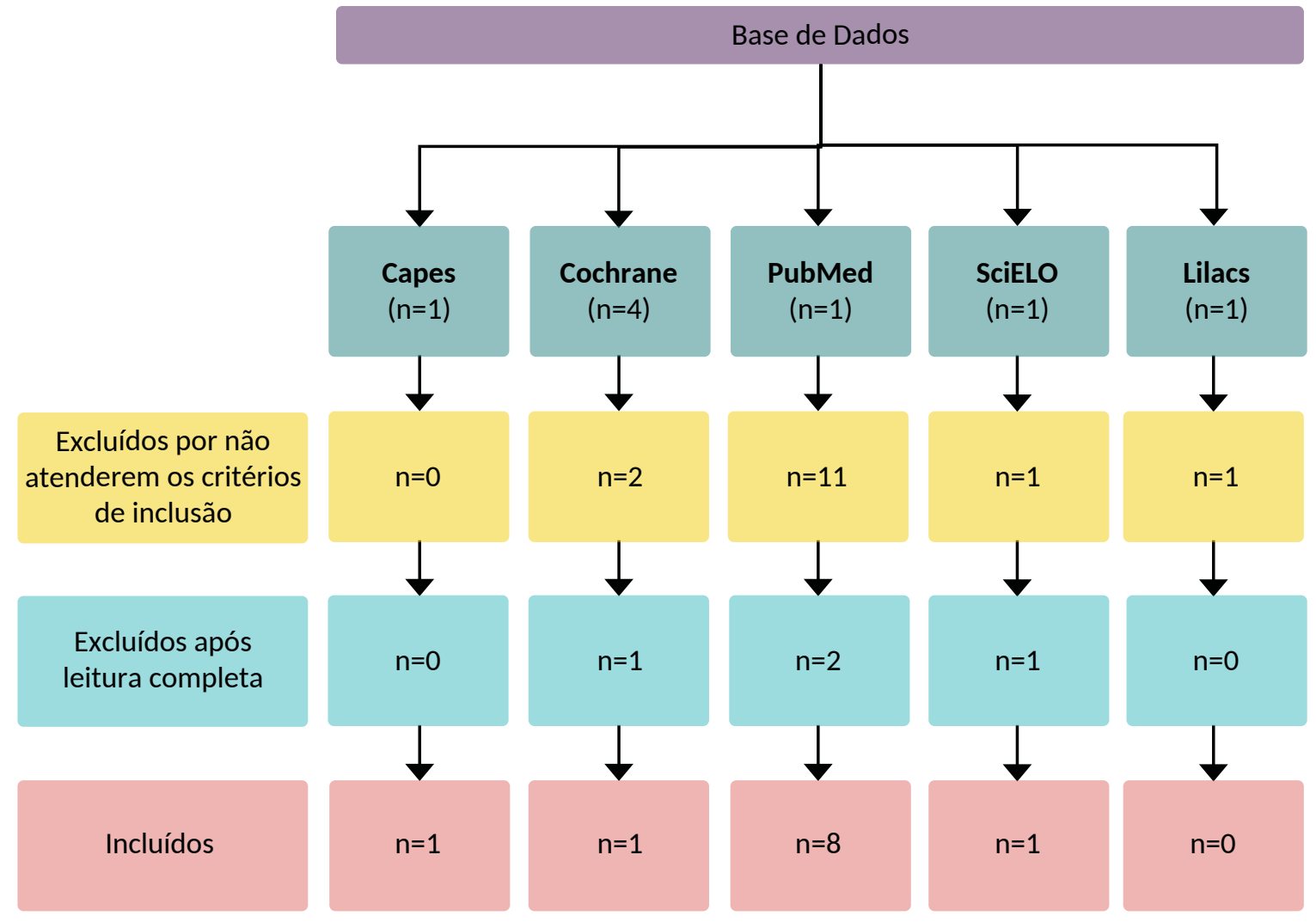

Quadro 1. Resumo sinóptico

\begin{tabular}{|c|c|c|c|c|}
\hline Título & $\begin{array}{l}\text { Autor(es); ano } \\
\text { de publicação }\end{array}$ & $\begin{array}{l}\text { País de publicação, } \\
\text { idioma }\end{array}$ & Metodologia & Objetivo \\
\hline $\begin{array}{l}\text { "Palliative care for people } \\
\text { with dementia" }\end{array}$ & $\begin{array}{l}\text { Sampson EL; } \\
2010^{17}\end{array}$ & Reino Unido, inglês & $\begin{array}{l}\text { Revisão } \\
\text { integrativa }\end{array}$ & $\begin{array}{l}\text { Revisar a literatura sobre } \\
\text { cuidados paliativos em } \\
\text { pacientes com demência. }\end{array}$ \\
\hline $\begin{array}{l}\text { "Cuidados paliativos } \\
\text { en personas con } \\
\text { demencia severa: } \\
\text { reflexiones y desafíos" }\end{array}$ & $\begin{array}{l}\text { Slachevsky } \\
\text { A, Abusleme } \\
\text { MT, Massa } \\
\text { AA; } 2016^{18}\end{array}$ & Chile, espanhol & $\begin{array}{l}\text { Revisão } \\
\text { narrativa }\end{array}$ & $\begin{array}{l}\text { Considerar os problemas } \\
\text { gerados por terapias utilizadas } \\
\text { em pacientes com demência } \\
\text { grave, analisando se são fúteis } \\
\text { ou excessivas no contexto. }\end{array}$ \\
\hline $\begin{array}{l}\text { "The role of gastrostomy } \\
\text { tube placement } \\
\text { in advanced dementia } \\
\text { with dysphagia: } \\
\text { a critical review" }\end{array}$ & $\begin{array}{l}\text { Goldberg LS, } \\
\text { Altman KW; } \\
2014^{19}\end{array}$ & EUA, inglês & $\begin{array}{l}\text { Revisão } \\
\text { sistemática }\end{array}$ & $\begin{array}{l}\text { Avaliar a literatura sobre } \\
\text { gastrostomia em pacientes com } \\
\text { demência avançada e disfagia. }\end{array}$ \\
\hline
\end{tabular}

continua... 
Quadro 1. Continuação

\begin{tabular}{|c|c|c|c|c|}
\hline Título & $\begin{array}{l}\text { Autor(es); ano } \\
\text { de publicação }\end{array}$ & $\begin{array}{c}\text { País de publicação, } \\
\text { idioma }\end{array}$ & Metodologia & Objetivo \\
\hline $\begin{array}{l}\text { "Consensos e dissensos na } \\
\text { indicação e continuidade } \\
\text { da terapia nutricional } \\
\text { enteral nos cuidados } \\
\text { paliativos de pacientes } \\
\text { com doenças crônicas } \\
\text { não transmissíveis" }\end{array}$ & $\begin{array}{l}\text { Castro JMF, } \\
\text { Frangella VS, } \\
\text { Hamada MT; } \\
2017^{20}\end{array}$ & Brasil, português & $\begin{array}{l}\text { Revisão } \\
\text { narrativa }\end{array}$ & $\begin{array}{l}\text { Apresentar consensos e } \\
\text { dissensos sobre a indicação } \\
\text { e continuidade da terapia } \\
\text { nutricional enteral nos } \\
\text { cuidados paliativos de } \\
\text { pacientes com doenças } \\
\text { crônicas. }\end{array}$ \\
\hline $\begin{array}{l}\text { "Artificial nutrition and } \\
\text { hydration in people with } \\
\text { late-stage dementia" }\end{array}$ & $\begin{array}{l}\text { Smith L, } \\
\text { Ferguson R; } \\
2017^{21}\end{array}$ & EUA, inglês & $\begin{array}{l}\text { Revisão } \\
\text { narrativa }\end{array}$ & $\begin{array}{l}\text { Examinar evidências } \\
\text { relacionadas ao uso de } \\
\text { nutrição e hidratação enteral } \\
\text { para pacientes com demência } \\
\text { em estágio avançado. }\end{array}$ \\
\hline $\begin{array}{l}\text { "Feeding decisions in } \\
\text { advanced dementia" }\end{array}$ & $\begin{array}{l}\text { Harwood RH; } \\
2014^{22}\end{array}$ & Reino Unido, inglês & $\begin{array}{l}\text { Revisão } \\
\text { narrativa }\end{array}$ & $\begin{array}{l}\text { Apresentar a influência da } \\
\text { nutrição e da hidratação } \\
\text { artificial na demência } \\
\text { avançada e aspectos éticos e } \\
\text { legais a se considerar para uma } \\
\text { melhor tomada de decisão. }\end{array}$ \\
\hline $\begin{array}{l}\text { "Enteral tube feeding } \\
\text { for older people with } \\
\text { advanced dementia" }\end{array}$ & $\begin{array}{l}\text { Sampson } \\
\text { EL, Candy } \\
\text { B, Jones L; } \\
2009^{23}\end{array}$ & Reino Unido, inglês & $\begin{array}{l}\text { Revisão } \\
\text { sistemática }\end{array}$ & $\begin{array}{l}\text { Avaliar resultados da terapia } \\
\text { nutricional enteral em idosos } \\
\text { com demência avançada } \\
\text { que desenvolvem problemas } \\
\text { com a deglutição ou } \\
\text { apresentam baixa ingestão } \\
\text { alimentar e nutricional. }\end{array}$ \\
\hline $\begin{array}{l}\text { "Ethical issues in } \\
\text { artificial nutrition and } \\
\text { hydration: a review" }\end{array}$ & $\begin{array}{l}\text { Geppert } \\
\text { CMA, } \\
\text { Andrews MR, } \\
\text { Druyan ME; } \\
2010^{24}\end{array}$ & EUA, inglês & $\begin{array}{l}\text { Revisão } \\
\text { narrativa }\end{array}$ & $\begin{array}{l}\text { Avaliar a nutrição e a } \\
\text { hidratação artificial em doenças } \\
\text { ameaçadoras da vida, como } \\
\text { demência avançada, e oferecer } \\
\text { orientações para instituições } \\
\text { formuladores de políticas e } \\
\text { profissionais da nutrição. }\end{array}$ \\
\hline $\begin{array}{l}\text { "Nasogastric feeding at } \\
\text { the end of life: a virtue } \\
\text { ethics approach" }\end{array}$ & $\begin{array}{l}\text { Krishna L; } \\
2015^{25}\end{array}$ & Singapura, inglês & $\begin{array}{l}\text { Revisão } \\
\text { narrativa }\end{array}$ & $\begin{array}{l}\text { Revisar o impacto ético e } \\
\text { clínico da terapia nutricional } \\
\text { enteral e fornecer informações } \\
\text { para a tomada de decisões nos } \\
\text { cuidados paliativos. }\end{array}$ \\
\hline $\begin{array}{l}\text { "Comfort feeding only: } \\
\text { a proposal to bring } \\
\text { clarity to decision- making } \\
\text { regarding difficulty with } \\
\text { eating for persons with } \\
\text { advanced dementia" }\end{array}$ & $\begin{array}{l}\text { Palecek EJ } \\
\text { et al.; } 2010^{26}\end{array}$ & EUA, inglês & Manuscrito & $\begin{array}{l}\text { Incentivar outros métodos } \\
\text { de alimentação que não } \\
\text { utilizem passagem de sonda } \\
\text { nasogástrica ou gastrostomia, } \\
\text { como alimentação via oral de } \\
\text { conforto, como alternativa que } \\
\text { traga maior qualidade de vida. }\end{array}$ \\
\hline $\begin{array}{l}\text { "Sobrevida e complicações } \\
\text { em idosos com doenças } \\
\text { neurológicas em } \\
\text { nutrição enteral" }\end{array}$ & $\begin{array}{l}\text { Martins AS, } \\
\text { Rezende NA, } \\
\text { Torres G; } \\
2012^{27}\end{array}$ & Brasil, português & $\begin{array}{l}\text { Estudo } \\
\text { prospectivo } \\
\text { observacional }\end{array}$ & $\begin{array}{l}\text { Avaliar as complicações e a } \\
\text { sobrevida de pacientes idosos } \\
\text { com doenças neurológicas em } \\
\text { uso de nutrição enteral. }\end{array}$ \\
\hline
\end{tabular}




\section{Discussão}

A revisão teve como objetivo verificar na literatura recente a utilização de terapia nutricional enteral em pacientes com demência em cuidados paliativos, visto que se trata de doença crônica ameaçadora de vida, e a nutrição é um marcador de prognóstico nesses pacientes. Para facilitar a interpretação e a discussão, os resultados foram divididos em três temas centrais: "cuidados paliativos e plano de cuidados", "cuidados paliativos e nutrição enteral" e "nutrição enteral em idosos com demência".

\section{Cuidados paliativos e plano de cuidados}

Como mencionado anteriormente, a demência é caracterizada por um declínio cognitivo progressivo, que leva a perda de funcionalidade e a torna uma doença crônica incurável, aumentando a necessidade de tratamento não curativo com abordagem paliativa. $O$ objetivo dos cuidados paliativos é melhorar a qualidade de vida, manter a funcionalidade e maximizar o conforto do paciente. Tais cuidados devem ser considerados durante toda a trajetória da doença, que se desenvolve em fases com diferentes níveis de funcionalidade 5,12,17-20.

$\mathrm{Na}$ demência avançada, por exemplo, há dependência em atividades básicas da vida diária, comunicação verbal limitada ou ausente, incapacidade de reconhecer familiares e problemas com apetite ou deglutição que dificultam ou impossibilitam a alimentação ${ }^{17,21,22}$. No entanto, Sampson, Candy e Jones ${ }^{23}$ ressaltam que não há consenso na literatura sobre o que define demência avançada ou em fase final, embora muitas das práticas clínicas sejam definidas de acordo com o estágio da doença.

De acordo com Harwood ${ }^{22}$, o plano de cuidados deve ser muito claro e estabelecido previamente com o paciente e seus familiares, para que todas as intervenções tenham embasamento ético, mesmo porque a tomada de decisão é muito mais difícil no estágio avançado da doença, quando a capacidade de consentimento do paciente acaba se perdendo. A aceitação do diagnóstico da demência e o conhecimento sobre sua evolução natural são importantes para que o paciente se prepare, elaborando um plano de cuidados ou diretivas antecipadas de vontade ${ }^{5}$. Cabe à equipe multiprofissional ou ao profissional médico que acompanha o caso esclarecer dúvidas, explicar o prognóstico da doença e mostrar alternativas para melhorar a qualidade de vida do paciente com demência, preservando sua autonomia e o direito de ser protagonista das próprias escolhas.

\section{Cuidados paliativos e nutrição enteral}

Para o ser humano, alimentar-se é muito mais do que ingerir nutrientes como meio de subsistência, para regenerar órgãos e tecidos e manter a vida. A alimentação envolve memórias afetivas, interações sociais, religião, cultura e hábitos, o que a torna um tema complexo e bastante controverso quando abordado em relação com os cuidados paliativos ${ }^{5,12,20}$.

$\mathrm{Na}$ assistência paliativa, a nutrição é definida de acordo com os objetivos do cuidado e a evolução da doença de base. Ela é considerada um importante fator, que auxilia o tratamento ao retardar comprometimentos funcionais e eventos adversos. Quando a enfermidade já não pode ser controlada, passa-se a priorizar o conforto, a qualidade de vida e o alívio dos sintomas. À medida que a doença avança, alguns indivíduos perdem a autonomia para alimentação, e a nutrição enteral pode ser instaurada ${ }^{5,20,24}$.

Muitos fatores estão ligados à opção pela nutrição enteral nos cuidados paliativos, a maioria deles relacionados ao medo da abstinência alimentar e ao sofrimento que ela pode causar no paciente e em seus familiares, visto que a alimentação é considerada símbolo de cuidado e conforto. Sua retirada, juntamente com a progressão da doença, ocasiona perda de peso, com impacto em condições físicas, clínicas e psicossociais, comprometendo a qualidade de vida do indivíduo ${ }^{20,23-26}$.

O objetivo da passagem de sonda ou ostomia - procedimentos para alimentação enteral é evitar pneumonias, aspiração e consequências da desnutrição, como lesões por pressão e infecções, prolongando a vida ao corrigir a desnutrição ${ }^{23,25}$. Entretanto, à medida que a doença avança e o paciente vai chegando à fase final da vida, o objetivo não deve ser mais a adequação 
nutricional, mas sim o conforto e alívio dos sintomas, ainda que isso signifique deixar o paciente permanecer em jejum ${ }^{5,12,20,24}$.

Dos artigos analisados, os principais achados estão relacionados com a ineficiência da nutrição enteral, que não tem aumentado a sobrevida ou gerado benefícios como evitar lesões por pressão, diminuir risco de aspiração e otimizar qualidade de vida e conforto ${ }^{18-26}$. A retirada ou suspensão do suporte nutricional se torna um dilema ético, uma vez que familiares, sem participar da discussão com a equipe médica, assistem à implementação da alimentação artificial no paciente com demência avançada acreditando que essa é a terapêutica adequada. Decisões tomadas desse modo vão contra os princípios de autonomia, beneficência e não maleficência e os ideais dos cuidados paliativos, que priorizam a qualidade de vida e consideram os desejos e valores do enfermo ao definir as decisões clínicas 5,20,21,24,25.

\section{Nutrição enteral em idosos com demência}

Entre as complicações mais comuns de pacientes com demência, principalmente em estágio mais avançado, estão problemas relacionados com alimentação: disfagia, aspiração do conteúdo alimentar, incapacidade para comer sozinho, resistência em se alimentar, dificuldade para mastigar ou utilizar utensílios e mudanças no apetite ${ }^{5,17,22-24,26}$. A perda de peso devida à baixa ingestão alimentar é frequente, podendo ocorrer nos estágios iniciais da doença, antes mesmo do diagnóstico. Todavia, é preciso destacar que a diminuição da taxa metabólica basal reduz as necessidades calóricas desses pacientes. Além disso, como em outras condições terminais, espera-se que o doente coma menos como parte da progressão natural no fim da vida, podendo manter uma ingestão oral mínima, suficiente para proporcionar conforto $22,23,26$.

Como já mencionado, existem dois métodos alternativos de alimentação quando a via oral não é mais possível: a passagem de sonda enteral ou as ostomias (gastrostomia/jejunostomia). A decisão sobre utilizar ou não esses métodos é difícil, pois envolve aspectos emocionais e éticos da prática dos prestadores de cuidados. A adoção dos procedimentos muitas vezes se baseia mais em crenças individuais de profissionais de saúde e membros da família do que em evidências científicas ${ }^{17,21}$.

A introdução ou continuidade da nutrição enteral em pacientes com demência, apesar de apropriada em alguns casos, causa maior agitação, desconforto e necessidade de sedação ou de restrições no leito. Há efeitos colaterais como sobrecarga de líquidos e aumento da diurese (levando à necessidade de sondagem) e das secreções respiratórias e gástricas, que podem gerar náuseas e vômitos. Além disso, nas ostomias, o procedimento cirúrgico tem elevado risco pós-operatório, incluindo eventos como pneumonia por aspiração, perfuração esofágica, migração do tubo, hemorragia e infecção da ferida ${ }^{20,23,25}$.

De acordo com os artigos analisados, faltam dados que corroborem benefícios em longo prazo da nutrição enteral em pacientes com demência avançada. Ficou demonstrado que seu uso não evita pneumonia por aspiração, não melhora o estado nutricional e a cicatrização de lesões por pressão, não diminui a mortalidade nem melhora a qualidade de vida. Há também falta de dados sobre efeitos adversos dessa intervenção ${ }^{17-26}$.

Estudo prospectivo realizado no Brasil em 2012 avaliou as complicações e a sobrevida de pacientes idosos com doenças neurológicas em uso de nutrição enteral, com elevado grau de dependência. A maioria $(91,2 \%)$ apresentou algum tipo de complicação, como pneumonia, obstrução ou perda da sonda, diarreia, constipação, vômito, extravasamento periostomia, refluxo e miíase, com elevada taxa de óbito ao término do estudo ${ }^{27}$. Em revisão sistemática de Goldberg e Altman ${ }^{19}$, pessoas com gastrostomia tiveram $54 \%$ de mortalidade em um mês e $90 \%$ de mortalidade em um ano. Outros achados demonstram que a inserção de gastrostomia durante a hospitalização aumentou o risco de mortalidade ${ }^{24}$.

No entanto, a suspensão total da nutrição enteral pode gerar desconforto a familiares e profissionais, visto que não há evidências ou diretrizes para a continuidade ou suspensão da medida. As decisões são baseadas na vontade do paciente ou, quando este não tem condições de decidir por si próprio, na deliberação da equipe multiprofissional com o consentimento dos familiares ${ }^{20}$. Suspender a alimentação simplesmente restabelece o processo normal de morrer, que tem ele mesmo propriedades analgésicas e reconfortantes naturais ${ }^{25}$. 
Do ponto de vista legal e ético, um adulto com capacidade de decidir pode optar pela retirada ou cessação da nutrição enteral. Além disso, é um ato de humanidade permitir que a morte prossiga sem o fornecimento de nutrição/hidratação em pacientes terminais. A suspensão da nutrição ou da hidratação não é a causa da morte do paciente com demência avançada ou em fase final de vida, mas sim a doença de base ${ }^{24}$.

A via oral é a via preferencial, fisiologicamente adequada, para alimentação. Privar o paciente do paladar, da sensação de texturas e sabores diferentes e até mesmo da interação no momento das refeições -, diminui sua autoestima e dignidade ${ }^{5,18,20}$. Uma alternativa que fica entre a nutrição enteral e a suspensão da alimentação é a chamada "alimentação de conforto": por via oral, com dieta na consistência adequada, a fim de evitar risco de aspiração mas garantir que o paciente consiga sentir o gosto dos alimentos e tenha prazer em comer, mesmo na demência em estágio avançado ${ }^{18,19}$.

Deve-se visar o máximo de conforto ao paciente, com maneiras menos invasivas de fornecer nutrição e hidratação. Respeitar preferências alimentares, rotinas e horários fora do padrão, incluindo mais lanches e petiscos, pode ajudar nessa estratégia, visto que, nos estágios avançados da demência, noções de alimentação saudável não são tão importantes quanto preferências individuais de quantidade e prazer ${ }^{21,22,26}$.

Dessa forma, o cuidado centrado no paciente reduz o sofrimento e é uma oportunidade para atividade, inclusão, engajamento, experiência social e prazer. Discutir com os familiares o plano de cuidados, escrever diretivas antecipadas de vontade, preferências e desejos é a chave para construir uma comunicação aberta e uma relação confiável entre paciente, profissionais e familiares ${ }^{22}$.

\section{Considerações finais}

A presente revisão analisou a produção científica recente sobre nutrição enteral em pacientes com demência em cuidados paliativos. Em caso de ausência de diretivas antecipadas de vontade, diante da dificuldade em definir estratégias para o plano de cuidados, o estudo espera ter fornecido informações para a tomada de decisão baseada em evidências científicas, e não somente em crenças ou na atuação rotineira da equipe.

A nutrição enteral é parte de um conjunto de medidas sustentadoras da vida, essenciais para recuperação e manutenção do suporte nutricional do paciente. No entanto, conforme o objetivo terapêutico vai se alterando, a nutrição acaba se tornando medida fútil, principalmente nos cuidados ao fim da vida. É necessário avaliar os reais objetivos da conduta nutricional em relação ao estágio avançado da demência, visando melhorar a qualidade de vida do paciente e respeitar seus desejos e os desejos de seus familiares. A alimentação de conforto é uma alternativa viável, que busca manter o prazer da comida e a interação social durante as refeições.

Com o aumento da expectativa de vida da população idosa, o número de pacientes com demência tende a aumentar. Por isso é necessário educar cuidadores e familiares sobre aspectos clínicos e medidas de conforto, a fim de que os cuidados paliativos sejam aplicados desde o início da doença. A comunicação aberta e a construção de um relacionamento de confiança com os membros da família são fundamentais nesse processo. Os familiares devem estar presentes em todas as etapas do cuidado, contribuindo com a tomada de decisões centrada no paciente.

Estudos de revisão têm discutido as limitações desse tipo de intervenção, visto que idosos em fase avançada da doença seriam incapazes de expressar seus desejos. No entanto, mais recentemente, artigos de abordagem qualitativa, com estudos de casos, têm proposto ferramentas práticas de comunicação que podem ser utilizadas pelos profissionais junto aos responsáveis pelo idoso ${ }^{28}$.

As próprias características da temática, que se refere a pessoas idosas com demência avançada, com comprometimento cognitivo acentuado, sobre as quais recai grande estigma, são fatores que têm limitado a quantidade de estudos qualitativos no mundo - o que, por outro lado, justifica o grande número de revisões. No contexto brasileiro, são necessários mais estudos de revisão que fortaleçam a prática baseada em evidências e forneçam informações para a criação de diretrizes que norteiem a prática clínica, visto que o assunto ainda está cercado por muitos tabus e questões éticas, emocionais, religiosas e culturais. 


\section{Referências}

1. Burlá C, Camarano AM, Kanso S, Fernandes D, Nunes R. Panorama prospectivo das demências no Brasil: um enfoque demográfico. Ciênc Saúde Coletiva [Internet]. 2013 [acesso 6 abr 2021];18(10):2949-56. DOI: 10.1590/S1413-81232013001000019

2. Lebrão ML. O envelhecimento no Brasil: aspectos da transição demográfica e epidemiológica. Saúde Coletiva [Internet]. 2007 [acesso 6 abr 2021];4(17):135-40. Disponível: https://bit.ly/3f6ao5R

3. Gallucci Neto J, Tamelini MG, Forlenza OV. Diagnóstico diferencial das demências. Rev Psiquiatr Clín [Internet]. 2005 [acesso 6 abr 2021];32(3):119-30. DOI: 10.1590/S0101-60832005000300004

4. World Health Organization. Global action plan on the public health response to dementia 2017-2025 [Internet]. Geneva: WHO; 2017 [acesso 6 abr 2021]. p. 52. Disponível: https://bit.ly/3f6auuf

5. Carvalho RT, Souza MRB, Franck EM, Polastrini RTV, Crispim DH, Jales SMCP et al. Manual da residência de cuidados paliativos. Barueri: Manole; 2018.

6. Sociedade Brasileira de Geriatria e Gerontologia. I Consenso Brasileiro de Nutrição e Disfagia em Idosos Hospitalizados [Internet]. Barueri: Minha Editora; 2011 [acesso 6 abr 2021]. Disponível: https://bit.ly/3o46H4B

7. Sociedade Brasileira de Nutrição Parenteral e Enteral, Colégio Brasileiro de Cirurgiões, Sociedade Brasileira de Clínica Médica, Associação Brasileira de Nutrologia. Terapia nutricional para pacientes na senescência (geriatria) [Internet]. 2011 [acesso 6 abr 2021]. Disponível: https://bit.ly/3o1EU4Y

8. Gonçalves TJM, Horie LM, Gonçalves SEAB, Bacchi MK, Bailer MC, Barbosa-Silva TG et al. Diretriz Braspen de Terapia Nutricional no envelhecimento. Braspen J [Internet]. 2019 [acesso 6 abr 2021];34(2 Supl):2-58. Disponível: https://bit.ly/33rCXFI

9. Calixto-Lima L, Gonzalez MC. Nutrição clínica no dia a dia. Rio de Janeiro: Rubio; 2017.

10. Brasil. Ministério da Saúde. Resolução RDC $n^{\circ} 63$, de 6 de julho de 2000. Diário Oficial da União [Internet]. Brasília, n. 130-E, p. 89-99, 7 jul 2000 [acesso 9 maio 2021]. Disponível: https://bit.ly/3bejVH2

11. Druml C, Ballmer PE, Druml W, Oehmichen F, Shenkin A, Singer P et al. ESPEN Guideline on ethical aspects of artificial nutrition and hydration. Clin Nutr [Internet]. 2016 [acesso 6 abr 2021];35(3):545-56. DOI: 10.1016/j.clnu.2016.02.006

12. Carvalho RT, Parsons HA, editores. Manual de Cuidados Paliativos ANCP [Internet]. $2^{\mathrm{a}}$ ed. São Paulo: ANCP; 2012 [acesso 6 abr 2021]. Disponível em: https://bit.ly/3uxTILe

13. Sociedade Brasileira de Geriatria e Gerontologia, Academia Nacional de Cuidados Paliativos. Vamos falar de cuidados paliativos [Internet]. Rio de Janeiro: SBGG; 2015 [acesso 6 abr 2021]. Disponível: https://bit.ly/3hfQ44K

14. Hopia H, Latvala E, Liimatainen L. Reviewing the methodology of an integrative review. Scand J Caring Sci [Internet]. 2016 [acesso 6 abr 2021];30(4):662-9. DOI: 10.1111/scs.12327

15. Mendes KDS, Silveira RCCP, Galvão CM. Revisão integrativa: método de pesquisa para a incorporação de evidências na saúde e na enfermagem. Texto Contexto Enferm [Internet]. 2008 [acesso 6 abr 2021];17(4):758-64. DOI: 10.1590/S0104-07072008000400018

16. Grupo Ânima Educação. Revisão bibliográfica sistemática integrativa: a pesquisa baseada em evidências [Internet]. Belo Horizonte: Grupo Ânima Educação; 2014 [acesso 6 abr 2021]. Disponível: https://bit.ly/2RFSoHC

17. Sampson EL. Palliative care for people with dementia. Br Med Bull [Internet]. 2010 [acesso 6 abr 2021];96(1):159-74. DOI: 10.1093/bmb/ldq024

18. Slachevsky A, Abusleme MT, Massa AA. Cuidados paliativos en personas con demencia severa: reflexiones y desafíos. Rev Med Chile [Internet]. 2016 [acesso 6 abr 2021];144:94-101. Disponível: https://bit.ly/3tvc9Pb

19. Goldberg LS, Altman KW. The role of gastrostomy tube placement in advanced dementia with dysphagia: a critical review. Clin Interv Aging [Internet]. 2014 [acesso 6 abr 2021];9:1733-9. DOI: 10.2147/CIA.S53153

20. Castro JMF, Frangella VS, Hamada MT. Consensos e dissensos na indicação e continuidade da terapia nutricional enteral nos cuidados paliativos de pacientes com doenças crônicas não transmissíveis. ABCS Health Sci [Internet]. 2017 [acesso 6 abr 2021];42(1):55-9. DOI: 10.7322/abcshs.v42i1.951 
21. Smith L, Ferguson R. Artificial nutrition and hydration in people with late-stage dementia. Home Healthc Now [Internet]. 2017 [acesso 6 abr 2021];35(6):321-5. DOI: 10.1097/NHH.0000000000000550

22. Harwood R. Feeding decisions in advanced dementia. J R Coll Physicians Edinb [Internet]. 2014 [acesso 6 abr 2021];44:232-7. DOI: 10.4997/JRCPE.2014.310

23. Sampson EL, Candy B, Jones L. Enteral tube feeding for older people with advanced dementia. Cochrane Database Syst Rev [Internet]. 2009 [acesso 6 abr 2021]. DOI: 10.1002/14651858.CD007209.pub2

24. Geppert CMA, Andrews MR, Druyan ME. Ethical Issues in artificial nutrition and hydration: a review. J Parenter Enter Nutr [Internet]. 2010 [acesso 6 abr 2021];34(1):79-88. DOI: 10.1177/0148607109347209

25. Krishna L. Nasogastric feeding at the end of life: a virtue ethics approach. Nurs Ethics [Internet]. 2015 [acesso 6 abr 2021];18(4):485-94. DOI: 10.1177/0969733011403557

26. Palecek EJ, Teno JM, Casarett DJ, Hanson LC, Rhodes RL, Mitchell SL. Comfort feeding only: a proposal to bring clarity to decision-making regarding difficulty with eating for persons with advanced dementia. J Am Geriatr Soc [Internet]. 2010 [acesso 6 abr 2021];58(3):580-4. DOI: 10.1111/j.1532-5415.2010.02740.x

27. Martins AS, Rezende NA, Torres G. Sobrevida e complicações em idosos com doenças neurológicas em nutrição enteral. Rev Assoc Méd Bras [Internet]. 2012 [acesso 6 abr 2021];58(6):691-7. DOI: 10.1590/ S0104-42302012000600014

28. Schwartz DB. Enteral nutrition and dementia integrating ethics. Nutr Clin Pract [Internet]. 2018 [acesso 6 abr 2021];33(3):377-86. DOI:10.1002/ncp.10085

Paula Abrantes Ciccarelli - Especialista - paula.cicca@gmail.com

(D) 0000-0003-0599-7076

Emanuela Bezerra Torres Mattos - Doutora - emattos@unifesp.br

(D) 0000-0002-1780-2355

Correspondência

Paula Abrantes Ciccarelli - Rua Professor Jose Leite e Oiticica, 434 apt. 608. Cep: 04505-080.

São Paulo/SP, Brasil.

Participação das autoras

Paula Abrantes Ciccarelli concebeu o projeto, interpretou os dados e redigiu o artigo.

Emanuela Bezerra Torres Mattos contribuiu com a interpretação dos dados e com a revisão crítica do conteúdo do artigo.

Recebido: 8.2.2021

Revisado: 3.5 .2021

Aprovado: 5.5.2021 